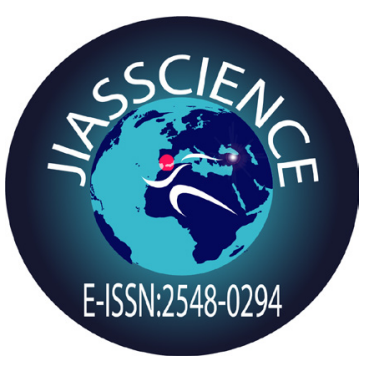

The Journal of International Anatolia Sport Science

Vol. 3, No. 3, December, 2018

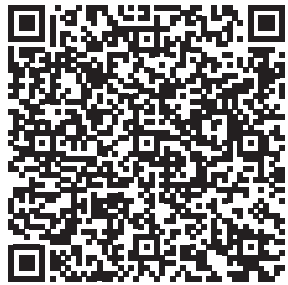

doi: $10.5505 /$ jiasscience. 2018.35229

\title{
NUTRITIONAL SUPPLEMENTS USE AND SOCCER PLAYERS' PERFORMANCE: A REVIEW OF THE LITERATURE
}

\author{
Mazin Abdul-Sattar ${ }^{1}$ \\ ${ }^{1}$ The Open Education College, Ministry of Education, Baghdad, Iraq
}

\begin{abstract}
The current usage of various nutrition supplements among soccer players has resulted in different consequences. The literature showed the effect of certain nutrition supplements intake on players' performance in a game play. This study therefore reviewed previous studies on the use of supplements intake among soccer players in order to understand the best- nutritional practices associated with better performance. A systematic review method was used to understand the current trends in using certain nutrition supplements and how such use can aid players' performance. The results showed that various supplements programs are followed to achieve certain outcomes. Findings from this study can be used to aid sport decision makers about the potential of certain nutrition supplements in developing soccer players.
\end{abstract}

Keywords: Nutrition Supplements, Soccer Performance, Soccer Development

\section{INTRODUCTION}

The progressive development in sport has led to uncertainty in terms of certain practices and its potential in aiding players' performance. This is mainly due to changes in games and play activities. The literature showed that various factors may potentially play a key role in the success of the player. This include the intake of nutrition which found to contribute in a small part to the development of players' genetic endowment, training, performance, and other factors. The nutritional intake among players can make the vital difference between winning and losing in according to the efforts spent by players to achieve their goals (Ruiz et al., 2005). In addition, different nutrition supplements are currently produced for increasing players' performance and abilities in the field (Leblanc, Gall, Grandjean, \& Verger, 2002). In complex team sports such as soccer, it is essential to understand how certain nutrition supplements intake can eventually influence players' performance in the field. This is without neglecting the diet and body building that are considered an essential part of soccer players. Despite the fact that most soccer players are following specific nutrition programs, still these programs may not necessarily contribute to our understanding of basic nutrition principles (Andrews \& Itsiopoulos, 2016). Moreover, the process associated with identifying the level of intake essential to maintain soccer players' needs may results in several consequences that are hard to dispel.

Therefore, previous studies have recommended several dietary and nutrition 
practices as an attempt to boost players' performance in the field. Yet, some of these practices may results in harmful consequences on the long run. This is where previous studies (e.g., Braun, von Andrian-Werburg, Schänzer, \& Thevis, 2018; Devlin, Leveritt, Kingsley, \& Belski, 2017) addressed the needs for better understanding of how certain nutrition supplements can be encouraged in sport settings.

The Football (soccer) activity is usually associated with the short sprints in an endurance context, particularly because it requires more efforts for coaches and soccer players to maintain skill levels throughout the duration of a game (Galanti et al., 2015). The distance covered for soccer players to advance through requires more efforts to be accomplished (estimated to be 8-13 $\mathrm{km}$ ). Thus, soccer players are required to consume certain energy levels estimated at a mean rate of $16 \mathrm{kcal} / \mathrm{min}$. This consumption is approximately relevant to oxygen consumption which correspond to $75 \%$ intake. As such, training soccer players requires special care and well-established nutrition program to keep them up to the level of competition, and numerous other factors. Nevertheless, preseason training is commonly practiced among soccer players where the work load reaches its highest peak, as players seek to achieve match fitness after the summer layoff (Spronk, Kullen, Burdon, \& O’Connor, 2014).

A well-designed nutrition supplements depends largely on the optimal style of players and training. For example, training time and effort spent by soccer players may requires certain types of supplements to provide their development needs. Therefore, this study aims at reviewing the current literature on nutrition supplements among soccer players and their impact on their performance in the field. This comes with understanding the consequences of such use in based on the integration of different substances. Despite the fact that some previous reviews on soccer players' development focused on the role of nutritional practices for enhancing performance, still less studies have been conducted to understand how certain nutritional supplements can affect players' performance. It is known that nutrition supplements that enhance some types of athletic performance may not necessarily enhance soccer players' performance. Thus, it is anticipated that outcomes from this work will provide the basis for understanding the different types of nutritional supplements and their impact on players' performance.

\section{METHODS}

This study is focused on one main research questions: What are the current use of nutritional supplements among soccer players? In order to better understand this aspect, this study attempted to examine the current literature in order to gather the required information for understanding the trends associated with nutritional supplement uses among players. The study protocol for searching the literature was shaped based on Tranfield, Denyer, and Smart (2003). Terms such as nutritional supplements, nutritional intake, nutrition for soccer, nutrition and soccer performance were commonly used in this review to refer to the use of nutritional supplements among soccer players in the field.

\section{Searching and screening}

The search of the databases was achieved by linking the mentioned topics above to the context of this study. For example, this study looked into sources of different online databases mainly to retrieve the required information from previously published articles. This include databases such as Cambridge Journals Online, Science Direct, Emerald, Oxford University Press (journals), Springer, and Taylor and Francis. The time of the search was not limited to certain periods. During the search, combinations of keywords was used, such as "nutritional supplements and soccer player performance" and "nutritional intake and development of players' performance". All the selected articles were scanned and assessed for eligibility for answering the research questions. This study applied some commonly used criteria to exclude studies that are not within the focus of this review.

1) Studies that are empirically oriented.

2) Focused on the role of nutritional supplement practices.

3) Involving soccer players. 
The searching of online databases resulted in 325 articles. However, only 33 articles were used in this study because they met all the inclusion criteria mentioned above (see Figure1).
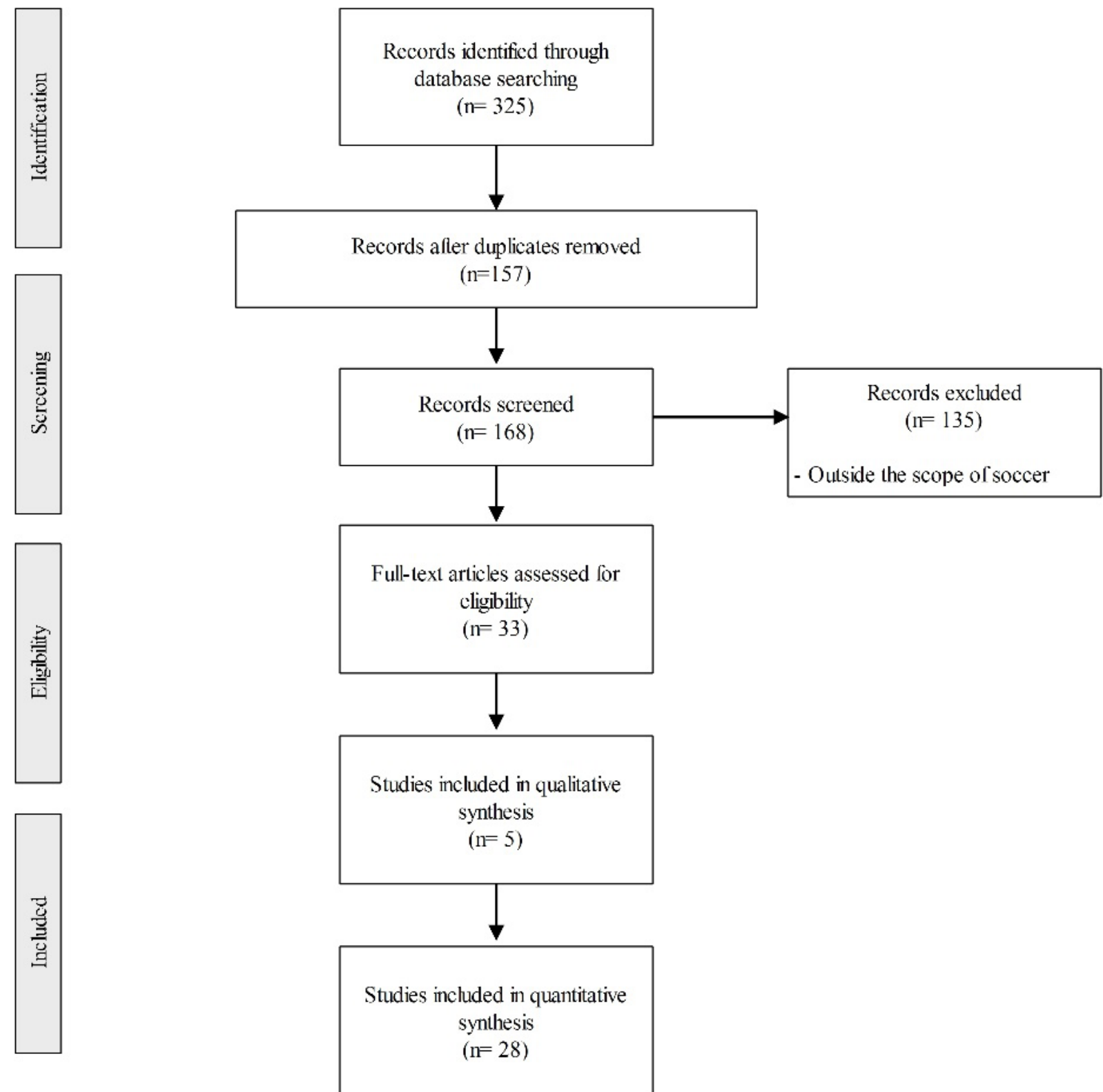

Fig 1. Flow diagram of article selection process

\section{RESULTS}

The search procedure mentioned above resulted in a number of articles related to the use of nutritional supplements in the soccer context. For example, a study by Lollo, Amaya-Farfan, and de Carvalho-Silva (2011) was conducted to determine the potential differences in the effect of three types of protein supplements on body composition, biochemical parameters and performance among soccer players in Brazil. The authors examined 24 athletes divided into three groups according to supplementation. All the participants received $1 \mathrm{gx}$ $\mathrm{kg}-1$ of body weight $\mathrm{x}$ day-1 of either whey protein (WP), hydrolyzed whey protein (HWP) or casein (CAS) after finishing the daily training design by the team's coach. Certain physical performance tests, and several biochemical variables in blood were used as an indication of changes in soccer players when taking these supplements. The results showed that there was no improvement in physical performance, but, casein supplementation 
resulted in muscle mass increase in which WP and HWP favoured the maintenance of the initial muscle mass. After all, the authors suggested that taking supplements after training sessions is still can be considered as a safe practice that can help sustaining muscle mass of players.

Tscholl, Junge, and Dvorak (2008), on the other hand, investigated the influence of using a proportion of nutritional supplements as part of medication in male top-level football players. Their study involved team physicians for assessing the level of influence of these supplements on the players' medication intake. A number of substances were related to nutritional supplements in which half of the players were found to take these at least once during a tournament and more than $10 \%$ prior to every match. The study found that such practices resulted in changing players' performance in the field.

As for examining changing in players' performance, Arent, Pellegrino, Williams, DiFabio, and Greenwood (2010) assessed how players' performance is changed among collegiate soccer players during preseason preparation. A total of 22 players were used to understand changes in their performance level during a progressive maximal treadmill test. The standard testing for baseline during training was used to randomly assigned to receive the nutraceutical blend $(\mathrm{n}=$ 12 ) or an isocaloric equivalent $(n=10)$ for 20 days of preseason training. Despite that the authors reported a noticeable improvement in soccer players, but there were no significant effects found from the use of supplementation on performance. The results also showed a significant decrease in the magnitude of the 8-isoprostane response throughout the training sessions. Preseason training in male college soccer players resulted in significant improvements in time-to-exhaustion. Supplementing with a proprietary antioxidant and nutraceutical blend were argued to promote players' performance based on the magnitude of the responses. Still, authors found some potential of using nutritional supplements on the reduction of stress among players.

Lee (2006) examined the use of nutritional supplements among elite soccer players in Korea which was categorized into health supplements, manufactured health food supplements, Chinese medicines and tonic foods, and nutrient supplements. A total of 241 players were participated in the study from different soccer clubs. The participants reported a use of $81.3 \%$ of nutritional supplements where health supplements were frequently reported followed by nutrient supplement drugs. Based on the survey results, the author found that supplements for weight gaining and calorie replacement were commonly taken by the players followed by vitamin supplements. The potential of these supplements was reflected in their training level and performance in which players with high nutrition intake reported higher age, duration of exercise and income than players with low nutrition intake. The decision for taking nutrition supplements among soccer players was found to be driven by family $(55.3 \%)$ or own decision (39\%), instead of coach's decision. The author suggested that the usage of nutritional supplements can offer a good potential for elite soccer players if proper use is considered.

Suzic Lazic et al. (2011) have conducted a mixed study (qualitative and quantitative) in order to determine the effect of dietary supplements and medication among elite athletes. The authors examined the effect of these supplements on 912 male players for a period of three days before training. The survey results showed that the majority of participants took medication of nonsteroidal anti-inflammatory drugs, while the rest used more than one drugs. Yet, the comparison of dietary supplements between players showed no differences. In addition, their study revealed widespread use of such types of supplements in which their effect on performance is still not clear or under researched. This is where the authors argued that potential effect can be found based on the amount, quantity and combination of the supplements.

Atashak and Jafari (2012) investigated the impact of using creatine (Cr) supplementation among soccer players. The study examined how $\mathrm{Cr}$ monohydrate (CrM) loading can play a role in increasing the serum enzymes activity and determining any short-term effect from taking $\mathrm{Cr}$ supplementation on serum lactate dehydrogenase (LDH), creatine phosphokinase (CK), and myocardial CK isoform (CKMB). The study was conducted on soccer players in which the results showed how serum $\mathrm{CK}$ and $\mathrm{CKMB}$ activity increases by the oral short-term CrM supplementation. Based on this, the authors argued that such intake of supplements among young players, especially CrM can result in significant effects on serum indirect markers of cellular damage.

Baralic et al. (2013) conducted a study on 
soccer player to assess the impact of astaxanthin (Asx) on paraoxonase (PON1) activities on soccer performance using certain supplements. The study consisted of 40 participants that were administrated to Asx and placebo (P) group. Physical and blood testing were taken before and after taking the supplements. Paraoxon and diazoxon were given to the participants undergoing the PON1 activity. The results showed that a significant interaction effect of supplementation and training on the players' performance in the training activity. However, there were no differences sported with regards to the use of these supplements in the P group. This is an indication that players may still find themselves to experience changes in performance of certain activities when certain supplements are taken.

Kreider (2003) conducted a systematics review of the literature on the use of ergogenic value of creatine supplementation and its impact on players' performance. The study examined how the use of creatine supplementation can contribute to the development of players' muscle physiology and/or exercise capacity. The author showed that most studies conducted on the use of creatine supplementation in short period of time can potentially increase creatine content by $10-30 \%$ and phosphocreatine stores by $10-40$. However, the author also reported that a potential effect of ergolytic can be found on players' performance in exercise. For instance, the process associated with short-term supplementation has been found to impose certain consequences on the player's performance, particularly by providing a maximal power/strength during sets of maximal effort muscle, as well as offering an additional support to the repetitive sprint performance.

Mohebbi, Rahnama, Moghadassi, and Ranjbar (2012) studied the impact of using creatine supplementation on soccer players' performance in the field. The study was conducted on a total of 17 players where they were administrated to either creatine or a placebo group. The repeated sprint test was used to assess changes among players when undertaking the two supplements reflected by the dribble and an accuracy of shooting to a target zone. The test results revealed a significant increase in the players' weight and lean body mass when using creatine only. In addition, the time of sprint running test and dribbling results were found to be significantly decreased in the creatine group.

Based on these, it can be said that the intake of certain supplements can significantly contribute to specific changes in players' performance while training.

\section{DISCUSSION AND CONCLUSION}

This review focused on the impact of certain nutritional supplements on soccer players' performance from different perspectives. It looked into the main dimensions that may influence players' development in a game play. The study found that aspects related to the players' characteristics such as age, weight, and training hours can significantly influence their performance while taking nutritional supplements. Findings from this study adds to the growing literature on the use of certain supplement and its impact on players' performance in the field. For example, this study adds to Burns, Schiller, Merrick, and Wolf (2004) who highlighted the needs to provide sound nutrition information, and provide services that meet the needs of a diverse population of student athletes. This study also supports the work of Manore, Patton-Lopez, Meng, and Wong (2017) which reported differences in nutrient requirements from certain athlete peers. They found that females would benefit from sport nutrition education that enhances food selection skills for health and sport performance. It also offers some bases to the work of Lohman, Carr, and Condo (2018) who reported that football players' nutritional intake is inconsistent with current recommendations for macronutrients in which players may benefit from additional knowledge on the risks and benefits of supplement use.

Creatine supplements were found to contribute to the players' weight and muscle increase with less impact on performance. This indicate that nutritional supplements may be useful for maintaining the physical outfit of the players, but not skills or performance. Therefore, more studies are needed to consolidate previous studies' findings and provide a more generalized view to the soccer context.

\section{REFERENCES}

Andrews, Michael C, \& Itsiopoulos, Catherine (2016) Room for improvement in nutrition knowledge and dietary intake of male football (soccer) players in Australia. International journal of sport nutrition and exercise metabolism 26(1) :55-64.

Arent, Shawn M, Pellegrino, Joseph K, Williams, Carey A, DiFabio, David A, \& Greenwood, John C. 
(2010) Nutritional supplementation, performance, and oxidative stress in college soccer players. The Journal of Strength \& Conditioning Research 24(4):1117-1124.

Atashak, S, \& Jafari, A. (2012) Effect of short-term creatine monohydrate supplementation on indirect markers of cellular damage in young soccer players. Science \& Sports 27(2): 88-93.

Baralic, Ivana, Djordjevic, Brizita, Dikic, Nenad, Kotur-Stevuljevic, Jelena, Spasic, Slavica, JelicIvanovic, Zorana, Pejic, Snezana (2013) Effect of astaxanthin supplementation on paraoxonase 1 activities and oxidative stress status in young soccer players. Phytotherapy research, 27(10): 1536-1542.

Braun, Hans, von Andrian-Werburg, Judith, Schänzer, Wilhelm, \& Thevis, Mario (2018) Nutrition Status of Young elite female German football players. Pediatric exercise science 30(1): 157-167.

Burns, Robert D, Schiller, M. Rosita, Merrick, Mark A, \& Wolf, Kay N. (2004) Intercollegiate student athlete use of nutritional supplements and the role of athletic trainers and dietitians in nutrition counseling. Journal of the American Dietetic Association 104(2): 246-249.

Devlin, Brooke L, Leveritt, Michael D, Kingsley, Michael, \& Belski, Regina. (2017). Dietary Intake, Body Composition, and Nutrition Knowledge of Australian Football and Soccer Players: Implications for Sports Nutrition Professionals in Practice. International journal of sport nutrition and exercise metabolism 27(2): 130-138

Galanti, Giorgio, Stefani, Laura, Scacciati, Irene, Mascherini, Gabriele, Buti, Gabriella, \& Maffulli, Nicola. (2015) Eating and nutrition habits in young competitive athletes: a comparison between soccer players and cyclists. Translational Medicine@ UniSa 11: 44 .

Kreider, Richard B. (2003) Effects of creatine supplementation on performance and training adaptations. Molecular and cellular biochemistry, 244(1-2): 89-94.

Leblanc, J Ch, Gall, F Le, Grandjean, V, \& Verger, Ph. (2002) Nutritional intake of French soccer players at the Clairefontaine training center. International Journal of Sport Nutrition and Exercise Metabolism, 12(3): 268-280.

Lee, HyunSook. (2006) The use of nutritional supplements in Korean elite soccer players. Korean Journal of Nutrition 39(3): 299-306.

Lohman, Rachel, Carr, Amelia, \& Condo, Dominique (2018) Nutritional Intake in Australian Football Players: Sports Nutrition Knowledge, Macronutrient and Micronutrient Intake. International journal of sport nutrition and exercise metabolism 1-21.

Lollo, Pablo, Amaya-Farfan, Jaime, \& de CarvalhoSilva, Luciano (2011) Physiological and physical effects of different milk protein supplements in elite soccer players. Journal of human kinetics 30: 49-57.

Manore, Melinda M, Patton-Lopez, Megan M, Meng, Yu, \& Wong, Siew Sun. (2017) Sport nutrition knowledge, behaviors and beliefs of high school soccer players. Nutrients 9(4): 350.

Mohebbi, H, Rahnama, N, Moghadassi, M, \& Ranjbar, K. (2012) Effect of creatine supplementation on sprint and skill performance in Young Soccer Players. MiddleEast J Sci Res 12(3): 397-401.

Ruiz, Fátima, Irazusta, Amaia, Gil, Susana, Irazusta, Jon, Casis, Luis, \& Gil, Javier (2005) Nutritional intake in soccer players of different ages. Journal of Sports Sciences 23(3): 235-242.

Spronk, Inge, Kullen, Charina, Burdon, Catriona, \& O'Connor, Helen. (2014) Relationship between nutrition knowledge and dietary intake. British Journal of Nutrition, 111(10): 1713-1726.

Suzic Lazic, J, Dikic, N, Radivojevic, N, Mazic, S, Radovanovic, D, Mitrovic, N, Suzic, S. (2011) Dietary supplements and medications in elite sportpolypharmacy or real need? Scandinavian journal of medicine \& science in sports 21(2): 260-267.

Tranfield, David, Denyer, David, \& Smart, Palminder (2003) Towards a methodology for developing evidence-informed management knowledge by means of systematic review. British journal of management 14(3): 207-222.

Tscholl, P, Junge, Astrid, \& Dvorak, Jiri (2008) The use of medication and nutritional supplements during FIFA World Cups 2002 and 2006. British Journal of Sports Medicine 42(9): 725-730. 